ments where there are known cases of infectious disease such as scarlet fever, should not be allowed to visit ; and the number of children in any ward at one time must be limited. Newborn babies should not be handled, and in many cases need not be shown to the child visitors at all. Only children of the mothers in the unit should be allowed to visit.

Despite the theoretical risks there is little evidence that visiting by children increases the incidence of infection, though few surveys have been made. P. S. Cook ${ }^{2}$ cited experience of a scheme whereby the mother's own children were allowed to visit; mothers and children were much happier, the staff derived satisfaction from seeing the obviously beneficial effects on the families, and there was no indication that the infection rate was increased. Pleuckhahn ${ }^{6}$ found no evidence of increased infection in the two-year period over which child visitors have been permitted at Geelong Hospital, Victoria. K. Vartan ${ }^{12}$ reported that no serious complications had arisen in five years' experience of child visiting. On balance it seems that visiting by children is not accompanied by a demonstrable rise in the sepsis rate ; and other objections such as inconvenience to the staff or complaints of noisy behaviour in the children can be overcome by good organization.

\section{Acute Optic Neuritis}

Acute optic neuritis is characterized by the onset of blurred vision, usually in one eye. The visual disturbance usually takes the form of a unilateral central scotoma, which gradually enlarges over a few days, persists for one to three weeks, and then gradually improves. The pupil on the affected side is usually dilated and its contraction to direct light is illsustained (Kestenbaum's sign). ${ }^{12}$

Over $80 \%$ of the patients with acute optic neuritis are between 20 and 50 years old, ${ }^{3}$ and the condition is twice as common in women as in men. The presenting symptom may be pain or tenderness in the eye, which is made worse by movement of the globe, particularly in a lateral direction. The pain is usually situated in or around the eye, the supraorbital region, or the frontal area, and it persists for several days and occasionally for as long as two or three weeks.

If no abnormality of the optic fundus is seen the condition is called "retrobulbar neuritis," but in nearly a quarter of cases there is some pinkness of the disc with blurring of its margins ("papillitis"). ${ }^{3}$ Sometimes the oedema of the disc is so severe that it resembles papilloedema. The oedema may spread to affect the retina and there may even be associated haemorrhages, though these are usually close to the disc margin (" neuroretinitis").

The differential diagnosis of unilateral central scotoma is sometimes difficult. Retinal vascular occlusion produces a more sudden onset, and the narrowed arteries (if the occlusion is arterial) or congested veins and haemorrhages (if venous)

\footnotetext{
Ruse F. C., Postgrad. med. F., 1964, 40, 692.
}

2 Rose, F. C., Proc. roy. Soc. Med., 1968, 61, 122.

sradley, W. G., and Whitty, C. W. M., F. Neurol. Neurosurg. Psychiat. $1967,30,531$.

Bıadley, W. G., and Whitty, C. W. M., f. Neurol. Neurosurg. Psychiat., $1968,31,10$.

- Rawson, M. D., Liversedge L. A., and Goldfast, G., Lancet, 1966, 2, 1344 . are easily seen. Vascular occlusion affecting the optic disc alone and temporal arteritis occur in an older age group. Compression of the optic nerve has a slower onset and the central scotoma eventually affects the periphery of the visual field as well. Toxic amblyopia (for example, due to tobacco) occurs in men after middle age, and the scotomata are bilateral and centrocaecal. Leber's hereditary optic atrophy is also bilateral, $80 \%$ of cases occurring in males usually between the ages of 10 and 30 ; there is a family history of the condition, and recovery to normal vision is rare.

In more than three-quarters of the cases of acute optic neuritis the visual acuity returns to normal or near normal within six months. ${ }^{3}$ Following recovery the patient may complain that colours appear much paler, but visual acuity is little affected and the only residual abnormality, which is not invariable, is temporal pallor of the optic disc.

Although many causes have been suggested for acute optic neuritis, the only definite one in some patients is multiple sclerosis. Of these patients $10 \%$ have evidence of demyelination elsewhere and about half will be found to have further episodes of demyelination if they are followed up for up to 20 years. Acute optic neuritis occurs in about half the patients with multiple sclerosis. In those patients who show no other signs of demyelination sinusitis has been suggested as a possible aetiological factor, but there is little evidence in favour of this."

Of the many remedies suggested, controlled trials showed that, though corticotrophin may speed recovery, it has no effect on either the eventual recovery of vision ${ }^{5}$ or on further attacks of demyelination.

\section{Disorders of Tyrosine Metabolism}

During the last decade reports have appeared with increasing frequency describing clinical disorders in children associated with abnormalities of tyrosine metabolism. The reader may easily become confused by them, since there is no general agreement on terminology.

Tyrosinosis (also called hereditary tyrosinaemia, tyrosinaemia, hepato-renal dysfunction) is a disorder first described by $\mathrm{K}$. Sakai and T. Kitagawa in $1957^{1}$ in a Japanese infant, and subsequently by others. ${ }^{2-4}$ It is inherited probably as an autosomal recessive condition. The syndrome includes cirrhosis of the liver and renal tubular defects, with vitaminD-resistant rickets, and is associated with a moderate rise in the serum tyrosine up to about $10 \mathrm{mg}$. per $100 \mathrm{ml}$. (normal up to $3 \mathrm{mg}$., depending on age and method) and tyrosyluria (which denotes excretion of tyrosine and its metabolites). A number of infants, particularly of French-Canadian origin, have had methioninaemia in addition ${ }^{5}$; these infants had a foul smell. The condition is associated with a deficiency of $p$-hydroxyphenylpyruvic acid oxidase ( $p$-H.P.P.A. oxidase) in the liver. ${ }^{6-9}$ The prognosis for untreated patients is poor. In some of them liver failure and death occur within the first 8 months or so of life, particularly if methioninaemia is present. ${ }^{4}$ Recent reports ${ }^{10-13}$ on the treatment of the condition by a diet low in tyrosine and phenylalanine, and in methionine too if necessary, are so encouraging that it should always be attempted in a properly diagnosed patient. Adequate facilities for controlling the diet and carrying out laboratory tests are essential. 